\title{
Ethanolic extract of Moringa oleifera leaves improve inflammation, angiogenesis, and blood pressure in rat model of preeclampsia
}

\author{
Agnes Batmomolin $^{1^{*}}$, Ahsan Ahsan ${ }^{2}$, I. Wayan Arsana Wiyasa ${ }^{3}$, Sanarto Santoso ${ }^{4}$ \\ ${ }^{1}$ Doctoral Program of Medical Science, Faculty of Medicine Universitas Brawijaya, Malang 65415, East Java, Indonesia. \\ ${ }^{2}$ Nursing Study Program, Faculty of Medicine, Universitas Brawijaya, Malang 65415, East Java, Indonesia \\ ${ }^{3}$ Department of Obstetrics and Gynecology Saiful Anwar Public Hospital, Malang, East Java, Indonesia \\ ${ }^{4}$ Department of Microbiology, Faculty of Medicine, Universitas Brawijaya, Malang 65145, East Java, Indonesia.
}

\begin{tabular}{l}
\hline ARTICLE INFO \\
\hline Received on: $16 / 02 / 2020$ \\
Accepted on: 14/05/2020 \\
Available online: 05/08/2020 \\
\hline Key words: \\
EMOL, IL-17, sFlt-1, \\
blood pressure, prevention \\
preeclampsia.
\end{tabular}

\begin{abstract}
The aim of this study is to investigate the effect of the ethanolic extract of Moringa oleifera leaves (EMOL) to improve inflammation, angiogenesis, and blood pressure by evaluating the serum level of IL-17, soluble vascular endothelial growth factor receptor 1 (sFlt-1), and blood pressure in a rat model of preeclampsia (PE). The rat model of PE was induced with N $\omega$-nitro-L-arginine methyl ester (L-NAME) of $50 \mathrm{mg} / \mathrm{kg}$ body weight (b.w) on day 12-18 of gestation. On day 5 of gestation, three groups are given EMOL dose of 50,100, and $200 \mathrm{mg} / \mathrm{kg}$ b.w, and one group administered aspirin of $1.35 \mathrm{mg} / 200 \mathrm{~g}$ b.w. Blood pressure was measurement on days 0,11 , and 19 of gestation. On day 20 of gestation, the rats were sacrificed, and serum parameters were analyzed. There is a significant difference in the serum level of IL-17 in all of the groups $(p<0.05)$. EMOL doses of 50 and $100 \mathrm{mg} / \mathrm{kg}$ b.w prevent an increase in the serum level of IL-17 better than low-dose aspirin $(p<0.05)$. There was a tendency decrease in sFlt-1 serum levels with EMOL doses of 50 and $100 \mathrm{mg} / \mathrm{kg}$ b.w, but statistically there are no significant differences $(p>0.05)$. All the doses of EMOL have the same effect as low-dose aspirin to prevent blood pressure increase in the rat model of $\mathrm{PE}(p<0.05)$.
\end{abstract}

\section{INTRODUCTION}

Preeclampsia (PE) is a core cause of maternal-fetal mortality worldwide (Weel et al., 2016). The main clinical manifestation of $\mathrm{PE}$ is high blood pressure, which can potentially cause a serious complication for maternal and fetus (Tannetta et al., 2013). The placenta is widely regarded as the core of PE growth. It started with a non-deep trophoblast attack, incomplete, and an imperfect remodeling of the spiral artery that causes immune imbalance, leading to the increased pro-inflammatory cytokine in chronic inflammation, autoantibodies to the angiotensin type 1 receptor (AT1-AA), and soluble vascular endothelial growth factor receptor 1 (sFlt-1) and the decreased nitric oxide (NO) (Cornelius, 2018).

\footnotetext{
${ }^{*}$ Corresponding Author

Agnes Batmomolin, Doctoral Program of Medical Science, Faculty of Medicine Universitas Brawijaya, Malang 65415, East Java, Indonesia. E-mail:agnesbat13@gmail.com
}

Interleukin 17 (IL-17) is one of the pro-inflammatory cytokines which plays a role in the expansion of systemic inflammation in PE in the maternal immune response to the allogeneic fetus. There is also an imbalance between angiogenesis and antiangiogenesis factor placental hypoxia due to an incomplete placental invasion in these diseases. This imbalance in PE pathophysiology is responsible for the increase of sFlt-1 (Huppertz, 2015; Raghupathy, 2013).

The only definitive treatment is delivery; therefore, prevention is the better way to decrease the impact of this disease. The low-dose aspirin has been used during pregnancy to prevent the onset of PE in women with a high risk of PE before week 16 of gestation and continued until the delivery (Gyamfi-Bannerman and Manuck, 2018).

Natural bioactive compounds have been known to provide endothelial protection through several mechanisms, such as promote the availability of eNOS/NO, mow down oxidative stress, inhibit NF-kB pathways, and decline the expression of cell adhesion molecules (Monsalve et al., 2017). Besides, mineral elements such as copper $(\mathrm{Cu})$ and manganese $(\mathrm{Mn})$ and Vitamins 
$\mathrm{D}, \mathrm{C}$, and $\mathrm{E}$ contribute to prevent the risk of PE (Azami et al., 2017; Karthivashan et al., 2016).

The leaves of Moringa oleifera has been shown to be rich in flavonoids, amino acid, multimineral vitamins, and other essential phytochemicals, which act as an antioxidant and antiinflammatory to treat hypertension. This can use as food and medicine. A study has been done on the composition of chemical compounds of the leaves of $M$. oleifera with various solvent forms including ether, water, methanol, and ethanol. The ether extract was only found in two compounds, water and methanolic extract consisted of seven compounds, whereas by using ethanol solvents, nine compounds were found: tannins, carbohydrates, saponins, glycosides, reduced sugars, steroids, terpenoids, flavonoids, and alkaloids. The most abundant of flavonoids is along with other phytochemical compounds (Nworu et al., 2013; Olson et al., 2016; Ugwu et al., 2013). Interestingly, flavonoids, especially quercetin, one of the compounds of extract of $M$. oleifera leaves (EMOL), are effective in all hypertension models, regardless of the origin of hypertension, the status of the renin-angiotensin system (RAS), oxidative stress, NO, and other factors. Even so, quercetin does not have a hypotensive effect on normotensive animal control (Perezvizcaino and Duarte, 2010). The ethanolic EMOL also contains high elements such as $\mathrm{Mn}$ and $\mathrm{Cu}$ that function as catalytic cofactor and activator antioxidant enzyme superoxide dismutase in chemistry redox of free radical scavenging (Karthivashan et al., 2016).

A research by (Owolabi et al., 2014) suggests that EMOL mitigates effect against lead toxicity in the cortex cerebral. $M$. oleifera as a dietary supplement in food can prevent nickel-induced nephrotoxicity in Wistar rat (Adeyemi and Elebiyo, 2014). As an antioxidant and a modulative influence effect on inflammatory cytokine, EMOL has a therapeutic effect against N-acetyl-paminophenol in kidney tissue (Karthivashan et al., 2016). EMOL was used extensively in the prevention or treatment of other disease but not in preventing PE. Therefore, this research aims to investigate the effect of EMOL on the prevention of PE by examining the serum level of IL-17, sFlt-1, and blood pressure in the rat model of PE.

\section{MATERIALS AND METHODS}

\section{Experimental design and animals}

This is an experimental in vivo study with a posttest only control group design. The study was conducted in the Laboratory of Bioscience Institute, Physiology Laboratory, and Biochemistry Laboratory, Universitas Brawijaya, Malang, Indonesia. All experiments in this study were carried out on 24 females Wistar rat weighing between 160 and $200 \mathrm{~g}$ of 12-16 weeks old $(n=4)$. The animals were obtained from Bioscience Institute Universitas Brawijaya, Malang, Indonesia. The animals were kept in the standardized animal cages, and the rats were fed with standardized rat pellets in free access to get food and water compliance with the Bioscience Institute of Universitas Brawijaya laboratory protocol (12-hour light-dark cycles, relative humidity, and stable temperature).

\section{Preeclampsia model and treatment}

All rats were acclimatized to laboratory condition for 1 week before the study. After a week of acclimatization, each female rat in the estrous cycle was housed with a male rat overnight to facilitate mating. The presence of spermatozoa plug in the vaginal lavage on the next day was counted as day 0 of gestation. On day 5 of gestation, rats were randomized into six groups $(n=4)$ : no treatment (control) group, $\mathrm{N} \omega$-nitro-L-arginine methyl ester (L-NAME) group that was only administered with L-NAME $50 \mathrm{mg} / \mathrm{kg}$ b.w, three groups administered with EMOL doses of 50,100 , and $200 \mathrm{mg} / \mathrm{kg} \mathrm{b.w}$, and one group which administered with aspirin dose of $1.35 \mathrm{mg} / 200 \mathrm{~g}$ b.w. The EMOL and aspirin were administered on day 5-18 of gestation, whereas PE-like rat model accepted L-NAME of $50 \mathrm{mg} / \mathrm{kg} \mathrm{b.w}$ for 7 days by gavage, similar to a previous study with modification in time of administering L.NAME starting on day 12-18 of gestation (Brown et al., 2013; Kaya et al., 2011).

\section{Preparation of EMOL}

Moringa oleifera leaf powder was obtained from Materia Medika, Batu, East Java, Indonesia. Then, the powder was extracted in the Department of Pharmacological, Universitas Brawijaya. Extraction process was performed using the maceration method with $90 \%$ ethanol, followed by evaporation to get the EMOL that are ready to use.

\section{Measurement of blood pressure}

On days 0,11 , and 19 of gestation, blood pressure (systolic and diastolic) of the rat was measured using volume pressure recording sensor by CODA non-invasive system from Kent Scientific Corporation (Wang et al., 2017).

\section{Measurement serum level of IL-17 and sFlt-1}

The rats were sacrificed using $40 \mathrm{mg} / \mathrm{kg}$ of intraperitoneal ketamine HCL and $1 \mathrm{mg} / \mathrm{kg}$ of xylazine hydrochloride on day 20 of gestation (Kaya et al., 2011). Blood was collected via the abdominal aorta in vacuum tubes without anticoagulant. Blood was centrifuged at 3,000 rpm for 20 minutes to get the serum and then stored at $-80^{\circ} \mathrm{C}$ before analysis. The serum level of IL-17 and sFlt-1/svascular endothelial growth factor receptor 1 measured by enzyme-linked immunosorbent assay kit was obtained from Elabscience, USA (catalog no: E-EL-RO566 for IL-17 and E-ELRO911 for sFlt-1). The procedure was carried out by following the instruction by a manufacturer.

\section{Statistical analysis}

The results of this study are expressed as mean \pm standard error of the mean. A statistical analysis was conducted using the SSPS software version 24.0 Normality test data were performed by using Shapiro-Wilk test, whereas homogeneity by using the Levene's test. The differences between the control and treatment groups were analyzed using a one-way analysis of variance, followed by post hoc LSD (least significance different). Data were considered to be statistically significant difference at $p$-value less than $0.05(p<0.05)$

\section{Ethical consideration}

This study was conducted in compliance with the principles expressed in the Declaration of Helsinki regarding ethical clearance from the Ethics Committee of the Faculty of Medicine, Universitas Brawijaya, Malang, Indonesia, No. 192/ EC/KEPK-S3/08/2018. 


\section{RESULTS}

\section{Ethanolic EMOL improves inflammation and angiogenesis in rat model of $P E$}

The serum IL-17 level is a pro-inflammatory cytokine measured in this study. We found that serum IL-17 levels in the group received EMOL doses of 50 and $100 \mathrm{mg} / \mathrm{kg} \mathrm{b.w} \mathrm{had} \mathrm{almost}$ the same effect as the control group, whereas the level of IL-17 in groups received EMOL doses of $200 \mathrm{mg} / \mathrm{kg}$ b.w and low-dose aspirin was closer to L-NAME/rat model of PE

Both the EMOL doses of 50 and $100 \mathrm{mg} / \mathrm{kg}$ b.w prevent the increase in the serum level of IL-17 better than low-dose aspirin (Fig. 1). There is a statistically significant difference in all groups that administered EMOL $(p<0.05)$. The groups received EMOL dose of 50 and $100 \mathrm{mg} / \mathrm{kg}$ b.w have significant differences compared to L-NAME group $(p<0.05)$.

Angiogenesis support in this study was measured by assessing the decrease in the serum levels of sFlt-1 or recognized as soluble fms-like tyrosine kinase (sFlt-1) that has antiangiogenic properties. The serum level of sFlt-1 is shown in Figure 2. The serum level of sFlt-1 in groups that received EMOL dose of 50 and $100 \mathrm{mg} / \mathrm{kg}$ b.w was almost the same of control group level. Hence, the serum level of sFlt-1 in EMOL dose of $200 \mathrm{mg} / \mathrm{kg}$ b.w group and low-dose aspirin group is almost the same as the group that received L-NAME to induce PE model, but statically there is no difference significant between all the groups $(p>0.05)$.

\section{Ethanolic EMOL improves blood pressure in rat model of PE}

The blood pressure was measured on day 0 and 11 of gestation to determine the initial blood pressure and the state of blood pressure before administering L-NAME to induce the rat model of $\mathrm{PE}$, whereas the measurement of the blood pressure on the day 19 of gestation was to evaluate the effect of L-NAME to induced rat model PE. Besides, the measurement of the blood pressure on day 19 of gestation was also to evaluate the effect both of EMOL and low-dose aspirin against blood pressure. This study result is shown in Figure 3.

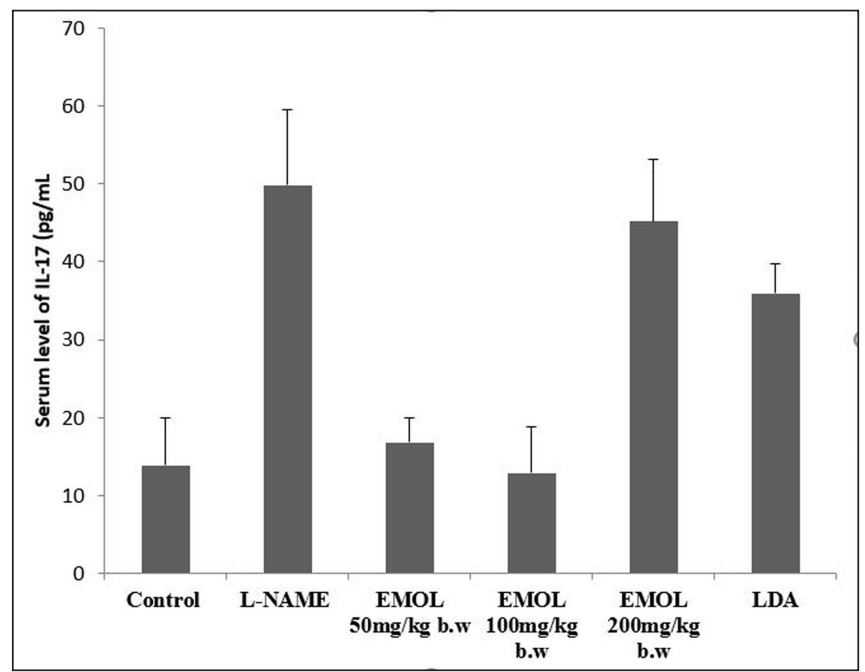

Figure 1. Effect of EMOL on serum level of IL-17 in rat model of PE. Value expressed as mean \pm SEM. Serum level of IL-17 significant different between all treatment $(p<0.05)$. EMOL 50 mg.kg b.w and 100 mg.kg b.w have significant different with L-NAME group $(p<0.05)$.
Blood pressure on day 0 and 11 of gestation within normal limits showed no increase, whereas both the systolic and diastolic blood pressures on day 19 of gestation showed an increase, especially in groups that administered L-NAME of 50 $\mathrm{mg} / \mathrm{kg}$ b.w on day 12 for 7 days, which were able to induce rat model of PE. The administered L-NAME dose of $50 \mathrm{mg} / \mathrm{kg} \mathrm{b.w}$ can induce the rat model of PE. This was revealed in the increase of blood pressure in L-NAME group compared to control group $(p<0.05)$.

In this study, we found that in all groups, administered EMOL and low-dose aspirin showed that there was no increase in both blood pressures: systolic and diastolic (Fig. 3). This means that EMOL has the effect of preventing an increase in blood pressure in the rat model of $\mathrm{PE}$, and they have statistically significant differences in all groups $(p<0.05)$. Interestingly, all the doses of EMOL 50, 100, and $200 \mathrm{mg} / \mathrm{kg}$ b.w have the same effect as the low-dose aspirin to prevent the increase both of systolic and diastolic blood pressures $(p<0.05)$.

\section{DISCUSSION}

IL-17 is a fascinating inflammatory biomarker that is in the center of discussion lately because it is a sensitive biomarker that takes a big part in the pathologic of PE. Serum IL-17 level rises in patients with PE compared with healthy pregnant and healthy women who are not pregnant (El Shahaway et al., 2019; Poordast et al., 2017).

The study shows that administering EMOL doses of 50 and $100 \mathrm{mg} / \mathrm{kg}$ b.w can prevent the increase in the serum IL-17 level, and its level on both doses is almost the same as the control group. The mechanism to explain how EMOL prevents the increase in the serum IL-17 level is through the pathway of NF- $\kappa B$. The NF$\kappa \mathrm{B}$ is the main pathway that regulates the inflammatory response through the production of cytokines and other inflammatory mediators, T-cell proliferation and differentiation, and dendritic cell maturation (Ginwala et al., 2019). Besides, hypoxia condition strengthens the NF- $\kappa \mathrm{B}$ pathway by increasing toll-like receptor

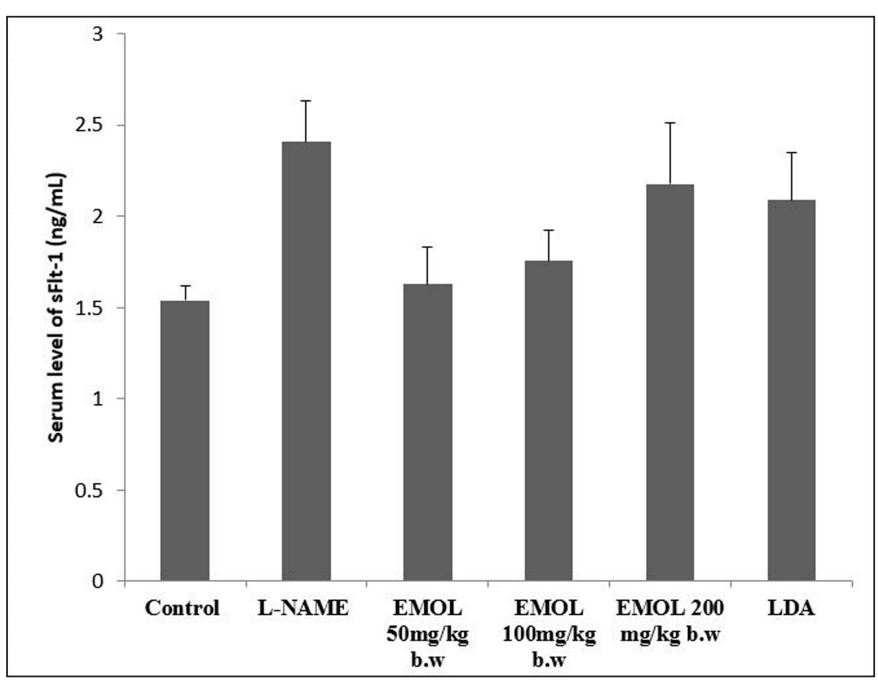

Figure 2. Effect EMOL on sFlt-1 level in rat model of PE. There in trend decrease of serum level sFlt-1 in EMOL $50 \mathrm{mg} / \mathrm{kg}$ and $100 \mathrm{mg} / \mathrm{kg}$ groups, but no Significant statistically $(p<0.05)$. 
A

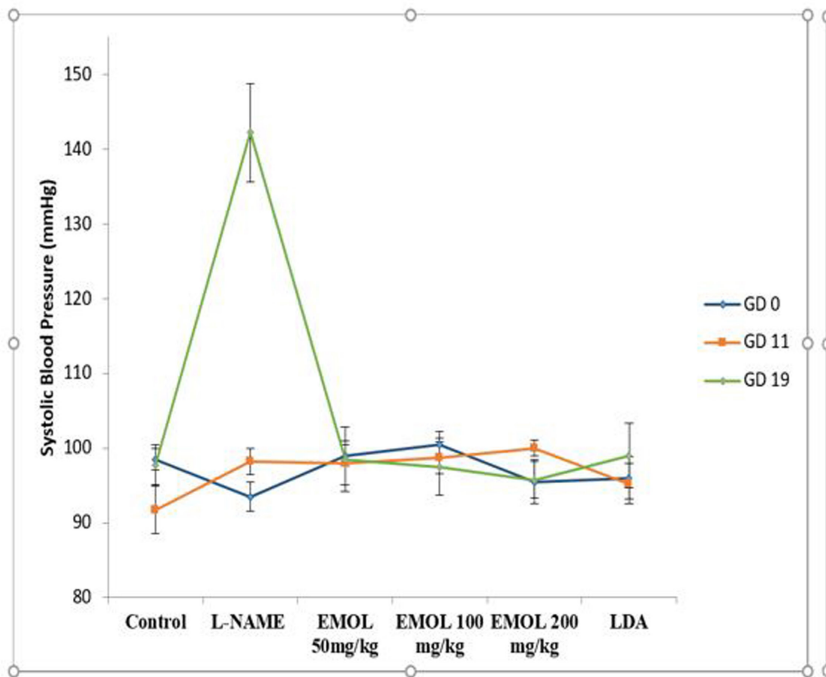

B

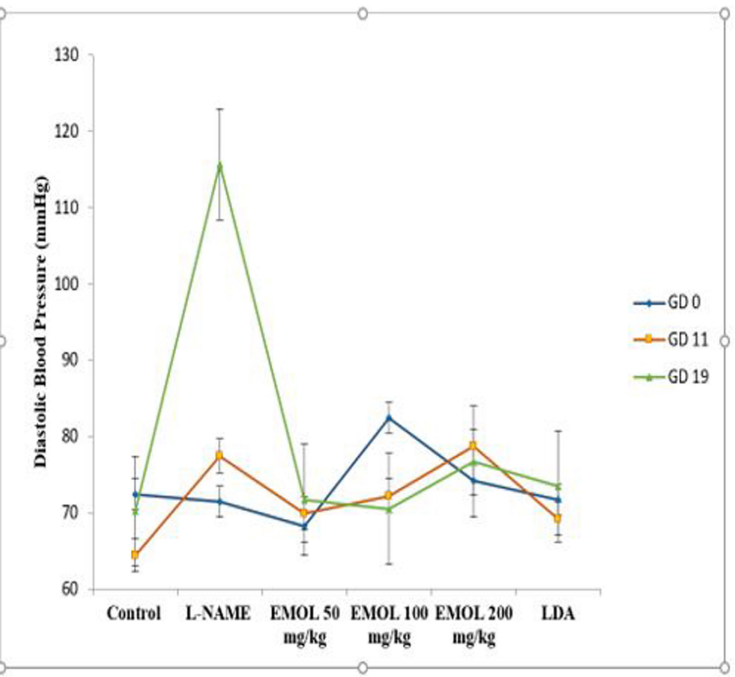

Figure 3. Blood pressure of the different groups on day 0,11 and 19 of gestation. A. Systolic blood pressure. B. Diastolic blood pressure, EMOL and LDA have the same effect to prevent increase of systolic blood pressure and diastolic blood pressure in rat model of PE $p<0.05$.

(TLR) expression and signaling, which increases the production of antimicrobial factors and stimulates phagocytosis, leukocyte recruitment, and adaptive immunity. Hypoxia-inducible factor $1 \alpha$ (HIF- $1 \alpha)$ and HIF-1 $\beta$ translocate to the nucleus, and they were binded as HIF to hypoxia response element, then inducing transcription genes including NF- $\mathrm{BB}$ and TLR in hypoxia condition (Schwartz et al., 2011).

Excessive systemic inflammatory responses, oxidative stress, and placental hypoxia in the progress of PE cause the activation of NF- $\kappa \mathrm{B}$ signaling and CD4 + differentiation, which, in turn, will increase IL-17 secretion by TH17, AT1-AA, and HIF-1 $\alpha$. An increased IL-17 can stimulate the production of other cytokines such as IL-6, IL-8, TNF- $\alpha$, granulocytes, and macrophage colony factors in PE (Cornelius, 2018; Fu et al., 2014). This condition worsens the development of this disease. Therefore, prevention is a better choice to reduce the fatal impact of PE which is maternal and fetal mortality.

The EMOL can inhibit the increase in the serum level of IL-17 in the rat model of PE because the active compounds of EMOL have anti-inflammatory activity (Molvarec et al., 2015; Pal et al., 2012). Anti-inflammatory bioactivity EMOL, which are flavonoid compounds, also work through an effective inhibition of IL-17A to their receptors (IL-17RA), which, in turn, causes the inhibition signaling of inflammation induced by TH17 cells that produce IL-17 (Arulselvan et al., 2016). The interaction between IL-17 and its receptors and NF-KB signaling is inhibited, and scavenging free radicals by EMOL flavonoids together with other pro-inflammatory cytokines and oxidative stress that worsens PE can be prevented.

Flavonoids can avoid cell damage by scavenging free radicals directly and thereby avoid their deleterious effects (Mahmoud et al., 2019). An increase in the serum level of IL-17 in EMOL dose of $200 \mathrm{mg} / \mathrm{kg} \mathrm{b.w}$ in groups in this study might have been because the cause of this dose is outside the effective dose for rat condition, and thus, it is a disadvantage.
The result of the serum levels of sFlt-1 in this study is shown in Figure 2. There is a significant difference in the level of sFlt-1 in L-NAME group compared to control group $(p<0.05)$. The serum level of sFlt- 1 in groups that administered EMOL dose of 50 and $100 \mathrm{mg} / \mathrm{kg} \mathrm{b.w} \mathrm{is} \mathrm{almost} \mathrm{the} \mathrm{same} \mathrm{as} \mathrm{control} \mathrm{group,}$ whereas in EMOL dose of $200 \mathrm{mg} / \mathrm{kg}$ b.w and low dose aspirin (LDA) approaches to the level of the L-NAME group, but there was no statistically significant difference. Although there is no statistically significant difference, there is a trend decrease of the serum level of sFlt-1 after administered EMOL. There are two possibilities for this result. First, sFlt-1 levels in the maternal circulation are reduced after Moringa leaf extract was given, but sFlt-1 was still produced and released by the placenta into the maternal circulation. Palmer et al. (2017) stated that the primary source of sFlt-1 is secretion of the placenta, which is secreted into the maternal circulation. Second, the prevention mechanism of increased blood pressure and excessive inflammation in a rat model of PE by EMOL does not occur via angiogenesis pathway and that is a need to be explored furthermore.

Ethanolic EMOL provides antihypertension in this study. EMOL prevents an increase in the systolic and diastolic blood pressure. Interestingly. EMOL and low-dose aspirin provide the same effect to prevent an increase in blood pressure. L-NAME has been observed to induce NO deficient, and a state of NO deficiency has been known to impair the relaxation vascular in human and animal models of PE. In this study, L-NAME was used to induce the rat model of PE (Korokin et al., 2011; Liu et al., 2017).

Hypertension is due in large part to increased oxidative stress, endothelin-1 overproduction, reduced production and/ or bioavailability of $\mathrm{NO}$, and/or RAS overstimulation. The antihypertensive effects of flavonoids are reduced oxidative stress, inhibition activity of angiotensin-converting enzyme, enhanced endothelial function, active effect on the vascular smooth muscle, and/or cell signaling modulation (Larson et al., 2010). Although we do not measure the mechanism of action of the bioactive 
compounds of EMOL in blood vessels such as the availability of eNO/NO, oxidative stress, and cell adhesion molecules in this study, we can argue that EMOL prevents an increase in blood pressure through the ability of the bioactive component EMOL, which maintains a balance of vasodilation and vasoconstriction factors of blood vessel (Amaral et al., 2017; Li et al., 2012).

Flavonoids also increase baroreflex sensitivity by reducing oxidative stress and modifying blood vessel elasticity and resistance, body fluid volume, autonomic nervous system, and angiotensin-renin system through anti-inflammatory and antioxidant activity (Monteiro et al., 2012).

EMOL containing essential elements such as $\mathrm{Cu}$ and $\mathrm{Mn}$ are also considered to be catalytic factors of the $\mathrm{Cu} / \mathrm{Zn}$-SOD and Mn-SOD antioxidant enzymes that can increase the activity of free radical scavenging and thus boost metabolism, whereas $\mathrm{Cu}$ is required for the metabolism of $\mathrm{Fe}$ and energy and can also act as a reduction agent in lysil oxidation enzymes cytochrome oxidase, superoxide dismutase, and dopamine hydroxylase (Karthivashan et al., 2016).

In this study, EMOL and low-dose aspirin are given early in pregnancy, on day 5 of gestation. Therefore, both EMOL and low-dose aspirin can provide an effect to reduce the risk factor in a rat model of PE by preventing an increase in the serum level of IL-17 and blood pressure. The low-dose aspirin was also known to more effectively reduce the risk of PE if used in early gestational age (before 16 weeks of pregnancy) until delivery (Roberge et al., 2016; Xu et al., 2015).

\section{CONCLUSION}

The ethanolic EMOL can improve inflammation and blood pressure by preventing increased serum level of IL-17 and blood pressure in the rat model of PE. EMOL also supports angiogenesis in a trend decrease of serum level of sFlt- 1 but not statistically different.

\section{ACKNOWLEDGMENTS}

This work supported by the Agency for Development and Empowerment of Health Professionals, Ministry of Health of the Republic of Indonesia.

\section{CONFLICT OF INTEREST}

Authors declared that there is no conflicts of interest.

\section{REFERENCES}

Adeyemi O. S., Elebiyo T. C. Moringa oleifera supplemented diets prevented nickel-induced nephrotoxicity in wistar rats. J Nutr Metab, 2014; 2014:1-8.

Amaral L. M., Wallace K., Owens M., LaMarca B. Pathophysiology and current clinical management of preeclampsia. Curr Hypertens Rep, 2017; 8:19-21.

Arulselvan P., Tan W. S., Gothai S., Muniandy K., Fakurazi S., Esa N. M., Alarfaj A. A., Kumar S. S. Anti-Inflammatory potential of ethyl acetate fraction of Moringa oleifera in downregulating the NF- $\kappa$ B signaling pathway in lipopolysaccharide-stimulated macrophages. Molecules, 2016; 21:1-14.

Azami M., Azadi T., Farhang S., Rahmati S., Pourtaghi K. The effects of multi mineral-vitamin D and vitamins $(\mathrm{C}+\mathrm{E})$ supplementation in the prevention of preeclampsia: an RCT. Int J Reprod Biomed, 2017; $5: 273-8$.
Brown C., Alexander-lindo R., Bishop K., Donovan D. The effects of S - nitrosoglutathione and S -nitroso- N - acetyl - D, L penicillamine in a rat model of pre - eclampsia. J Nat Sci Biol Med, 2013; $2: 330-5$.

Cornelius D. C. Preeclampsia: from inflammation to immunoregulation. Clin Med Insights Blood Disord, 2018; 11:1-6.

El Shahaway A. A., Elhady R. R. A., Abdelrhman A. A., Yahia S.. Role of maternal serum interleukin 17 in preeclampsia: diagnosis and prognosis. J Inflamm Res, 2019; 12:175-80.

Fu B., Tian Z., Wei H.. TH17 cells in human recurrent pregnancy loss and pre-eclampsia. Cell Mol Immunol, 2014; 11(6):564-70.

Ginwala R., Bhavsar R., Chigbu D. I., Jain P., Khan Z. K. Potential role of flavonoids in treating chronic inflammatory diseases with a special focus on the anti-inflammatory activity of apigenin. Antioxidants (Basel), 2019; 8(2):1-30.

Gyamfi-Bannerman C., Manuck T. ACOG committee opinion, low-dose aspirin use during pregnancy. Obstet Gynecol, 2018; 132:44-52.

Huppertz, B. Maternal-fetal interactions, predictive markers for preeclampsia, and programming. J Reprod Immunol, 2015; 108:26-32.

Karthivashan G., Kura A. U., Arulselvan P., Isa N. M., Fakurazi S. The modulatory effect of Moringa oleifera leaf extract on endogenous antioxidant systems and inflammatory markers in an acetaminopheninduced nephrotoxic mice model. PeerJ, 2016; 4:1-18.

Kaya A., Boztosun A., Seckin P. H., Guven S., Kucukdurmaz Z., Gulturk S., Cevit O. The evaluation of hypoxia-inducible factor 1 in model of pregnant rats. J Investig Med, 2011; 59:1268-72.

Korokin M. V, Pokrovsky M. V, Novikov O. O., Gureev V. V, Denisyuk T. A., Korokina L. V, Polyanskaya O. S., Ragulina V. A., Pokrovskaya T. G., Danilenko L. M., Belous A. S. Effect of L-Arginine, vitamin B 6 and folic acid on parameters of endothelial dysfunction and microcirculation in the placenta in modeling of L-NAME-induced no deficiency. Bull Exp Biol Med, 2011; 152:70-3.

Larson A. J., Symons J. D., Jalili T. Quercetin: a treatment for hypertension?-A review of efficacy and mechanisms. Pharmaceuticals, 2010; 3:237-50.

Li H., Andriantsitohaina R., Auger C., Chataigneau T., Nelly E., Martı M. C. Review article molecular mechanisms of the cardiovascular protective effects of polyphenols. Br J Nutr, 2012; 108(9):1532-49.

Liu C., Zhu L., Fukuda K., Ouyang S., Chen X., Wang C., Zhang C., Martin B., Gu C., Qin L., Rachakonda S., Aronica M., Qin J., Li X. The flavonoid cyanidin blocks binding of the cytokine interleukin-17A to the IL-17RA subunit to alleviate inflammation in vivo. Sci Signal, 2017; 10(467):eaaf8823.

Mahmoud A. M., Hernández Bautista R. J., Sandhu M. A., Hussein O. E. Beneficial effects of citrus flavonoids on cardiovascular and metabolic health. Oxid Med Cell Longev, 2019; 2019:1-19.

Molvarec A., Czegle I., Szijarto J., Rigo, J. Increased circulating interleukin-17 levels in preeclampsia. J Reprod Immunol, $2015 ; 112: 53-57$

Monsalve B., Concha-meyer A., Palomo I., Fuentes E. Mechanisms of endothelial protection by natural bioactive compounds from fruit and vegetables. An Acad Bras Cienc, 2017; 89:615-33.

Monteiro M. M. O., França-silva M. S., Alves N. F. B., Porpino S. K. P., Braga V. A. Quercetin improves baroreflex sensitivity in spontaneously hypertensive rats. Molecules, 2012; 12997-13008.

Nworu C. S., Okoye E. L., Ezeifeka G. O., Esimone C. O. Extracts of Moringa oleifera Lam. showing inhibitory activity against early steps in the infectivity of HIV-1 lentiviral particles in a viral vector-based screening. Afr J Biotechnol, 2013; 12:4866-73.

Olson M. E., Sankaran R. P., Fahey J. W., Grusak M. A., Odee D., Nouman, W. Leaf protein and mineral concentrations across the "Miracle tree" genus moringa. PLoS One, 2016; 11:1-17.

Owolabi J., William F., Olanrewaju J., Etibor T. A., Fabiyi O., Anglais A. E. Histomorphological evidences of Moringa oleifera' s 
ameliorative effects against lead toxicity in cerebral cortex. World J Life, 2014; 3:53-58.

Pal G., Singh G. P., Garg R., Sharma S. K. Antiinflammatory evaluation of leaf extract fo Moringa oleifera. J Pharm Sci Innov, 2012; $1: 22-24$.

Palmer KR, Tong S, Kaitu'u-Lino TJ. Placental-specific sFLT-1: role in pre-eclamptic pathophysiology and its translational possibilities for clinical prediction and diagnosis. Mol Hum Reprod, 2017; 23:69-78.

Perez-vizcaino F., Duarte J. Molecular aspects of medicine flavonols and cardiovascular disease. Mol Aspects Med, 2010; 31:478-94.

Poordast T., Najib F. S., Baharlou R., Bijani A., Alamdarloo S. M., Poordast A. Assessment of T helper 17-associated cytokines in third trimester of pregnancy. Iran J Immunol, 2017; 14:172-9.

Raghupathy, R. Cytokines as key players in the pathophysiology of preeclampsia. Med Princ Pract, 2013; 22:8-19.

Roberge S., Sibai B., McCaw-Binns A., Bujold E. Low-dose aspirin in early gestation for prevention of preeclampsia and small-forgestational-age neonates: meta-analysis of large randomized trials. Am J Perinatol, 2016; 33:781-5.

Schwartz R. S., Eltzschig H. K., Carmeliet P. Hypoxia and inflammation. N Engl J Med, 2011; 364:656-65.

Tannetta D. S., Dragovic R. A., Gardiner C., Redman C. W., Sargent, I. L.. Characterisation of syncytiotrophoblast vesicles in normal pregnancy and pre-eclampsia: expression of Flt-1 and endoglin. PLoS One, 2013; 8:e56754.
Ugwu O. P. C., Nwodo O. F. C., Joshua P. E., Bawa A., Ossai E. C., Odo C. E. Phytochemical and acute toxicity studies. Int J Life Sci Biotechnol Pharma Res, 2013; 2:66-71.

Wang Y., Thatcher S. E., Cassis, L. A. Chapter 6 measuring blood pressure using a noninvasive tail cuff method in mice. Methods Mol Biol, 2017; 1614:69-73.

Weel I. C., Baergen R. N., Rom M., Borges V. T., Ribeiro R., Witkin S. S., Bannwart-Castro C., Peraçoli J. C., De, L. Association between placental lesions, cytokines and angiogenic factors in pregnant women with preeclampsia. PLoS One, 2016; 11:1-16.

Xu T. T., Zhou F., Deng C. Y., Huang G. Q., Li, J. K., Wang, X. D. Low-dose aspirin for preventing preeclampsia and its complications: a meta-analysis. J Clin Hypertens, 2015; 17:567-73.

How to cite this article:

Batmomolin A, Ahsan A, Wiyasa IWA, Santoso S. Ethanolic extract of Moringa oleifera leaves improve inflammation, angiogenesis, and blood pressure in rat model of preeclampsia. J Appl Pharm Sci, 2020; 10(08):052-057. 